\title{
Characterization of "Super-Responders" to Cryoballoon Ablation
}

\author{
Martí-Almor J*, Casteigt B, J iménez-López J, \\ Conejos J, Vallès $E$ and Cladellas $M$ \\ Arrhythmia Unit, Cardiology Department, Hospital del \\ Mar, IMIM, UAB, Barcelona, Spain \\ *Corresponding author: J ulio Martí-Almor, Paseo \\ Marítimo de la Barceloneta 25-29, 08003, Barcelona, \\ Spain
}

Received: August 04, 2021; Accepted: September 03, 2021; Published: September 10, 2021

\begin{abstract}
Background: Pulmonary Veins Isolation (PVI) is the cornerstone in the treatment of atrial fibrillation. Recurrence rate is common in the long-term followup (FU); nevertheless, some patients maintain Sinus Rhythm (SR) for more than 5 years after the index ablation. The aim of this study is characterize this kind of patients so called "super-responders".
\end{abstract}

Methods: This is a retrospective single-center study including all patients summited for cryo-balloon ablation in our hospital from January 2011 to September 2020. We investigated clinical, electrocardiographic, echocardiographic variables and those linked to the ablation procedure. A univariate and multivariate logistic regression was performed.

Results: During this period, 422 patients underwent PVI; however, 193 were excluded: 21 lost in the FU, 30 got radiofrequency ablation and 142 did not reach a minimum 5 years FU. Of the 229 finally included, 85 (group 1) did not have any recurrence during the follow-up, in front of 144 (group 2) with AF recurrences. In the multivariate analysis the $p$ wave duration: OR: $0.92 ; 95 \%$ $\mathrm{Cl}(0.89-0.94) ; \mathrm{p}<0.001$, BMI kg/m: OR: 0.74; 95\% Cl (0.65-0.85); $\mathrm{p}<0.001$, a temperature $<-40^{\circ} \mathrm{C}$ in all the targeted veins: OR: $3.52 ; 95 \% \mathrm{Cl}(1.45-8.54)$; $\mathrm{p}=0.005$ and $\mathrm{SR}$ on the ablation index day OR: $7.29 ; 95 \% \mathrm{Cl}(1.53-34.71)$; $p=0.012$, maintained statistical significance.

Conclusions: In our series the $p$ wave duration, BMI, the presence of SR the ablation index day and achieving a temperature $<-40^{\circ} \mathrm{C}$ in all the targeted veins, resulted as protective factors to maintain $S R$ in the long term follow-up. An adequate selection of patients probably could improve results and optimize resources.

Keywords: Cryo-balloon ablation; Atrial fibrillation; Super-responders; Obesity; P wave duration

\section{Introduction}

Pulmonary Vein Isolation (PVI) is the cornerstone of symptomatic Atrial Fibrillation (AF) treatment and it has been proven clearly superior to Antiarrhythmic Drugs (AAD) either for paroxysmal or persistent presentation [1]. The energy source used to achieve isolation either point by point Radiofrequency Ablation (RFA) or single shot Cryo-Balloon Ablation (CBA) has shown a similar efficacy $[2,3]$, but the learning curve of the former is much slower than CBA. That simple fact contributed to the great spread of CBA all over the world.

Recurrence rate during the Follow Up (FU) after the blanking period is high $[1,4,5]$. Factors related to acute recurrence as advanced age, hypertension, obesity, enlargement of left atrial size, nonparoxysmal presentation and the presence of obstructive sleep apnea, have been extensively described [6]. However, few studies have focused in for long term FU recurrences ( $>5$ years) [4,5]. By the other hand, a non-negligible number of patients persist in Sinus Rhythm (SR) many years after the index PVI procedure, without any further recurrence.

The aim of this study is to characterize these so-called "super- responders" patients, defined by those in whom no recurrence have been detected after a minimum FU period of 5 years after the index ablation, comparing them to those with recurrences.

\section{Methodology}

\section{Study population and data collection}

This is a retrospective single-center study including all consecutive patients undergoing a CBA for PVI from January 2011 to September 2020. All patients signed an informed consent for the procedure approved by the local Ethics Committee in our center, and the institute's committee on human research has approved the study protocol. During this period both first-generation (CB1) and second-generation (CB2) cryo-balloon catheters (Medtronic inc. Minneapolis. USA) were used.

Data collection included clinical and demographic variables, as age, sex, history of hypertension, diabetes, coronary artery disease, $\mathrm{CHA}_{2} \mathrm{DS}_{2}$ VASc score, significant structural heart disease, Body Mass Index (BMI), presence of Obstructive Sleep Apnea (OSA). In this regard, a screening Polysomnography (PSG) was performed in all patients with a $\mathrm{BMI} \geq 30$ or if there were symptoms related with OSA. We also collected time from diagnosis to AF ablation, type 
of atrial fibrillation and patient rhythm the ablation index day Echocardiographic variables included Left Ventricular Ejection Fraction (LVEF), left atrial size (anteroposterior diameter in parasternal view). Electrocardiographic variables included $p$ wave duration in lead DIII in milliseconds (ms) and $\mathrm{p}$ wave amplitude in DII in millivolts $(\mathrm{mv})$. Both variables were obtained using a specific software (Philips Inc Eindhoven. NL) able to increase tenfold the EKG, using manual calipers by two experienced electrophysiologists. In case of discrepancy over $10 \mathrm{~ms}$ or $0.01 \mathrm{mv}$, a consensus was reached by the intervention of a third electrophysiologist. The EKG registries used for this study, were obtained after AAD withdrawal. Only in patients in AF in whom AF persisted in the first control after the blanking period, the measures were performed using the EKG recorded just after ablation.

Intra-procedure characteristics as anatomy of $\mathrm{PV}$, minimal temperature in all PV above or below $-40^{\circ} \mathrm{C}$, entrance/exit block of $\mathrm{PV}$, and cavotricuspid isthmus block if performed, were also collected.

\section{Ablation procedure}

CBA procedures were performed under conscious sedation. All patients had a previous imaging technique (computed tomography, magnetic resonance or rotational angiography) to assess left atrium and PV anatomy. All procedures were guided by intracardiac echocardiography, and since 2013, all procedures were monitored with an esophageal temperature probe. After having achieved left atrial access with a single transeptal puncture a $100 \mathrm{IU} / \mathrm{kg}$ heparin bolus was given and a continuous perfusion is maintained through the Flex Cath sheath (Medtronic Inc) in order to obtain an ACT between 250-300 seconds. A 20mm diameter Achieve inner lumen-mapping catheter (Medtronic Inc) was sequentially positioned in the ostium of each PV to obtain baseline electrical information. Then a $28 \mathrm{~mm}$ double-walled cryoballoon (Arctic Front or Arctic Front Advance. Medtronic Inc) was advanced over the Achieve catheter, inflated and positioned in the PV ostium of each vein. Optimal vessel occlusion was considered to have been achieved when selective die injection showed total contrast retention with no back flow to the atrium. For the right side, PV a continuous pacing with a tetrapolar catheter placed in the superior vena cava was used to pace the phrenic nerve to avoid phrenic nerve palsy. Application times varied along time, in the first-generation balloon, we performed at least two applications per vein, over 300 seconds each, with the second-generation balloon, we performed two applications over 240 seconds each, since 2018 we moved to a double 180 seconds application. Extra bonus application was performed in any case if PV potentials remain visible. Twenty minutes after performing the last application, we rechecked entrance and exit block of PVI repositioning the Achieve catheter in all the veins. If reconnections were detected, extra applications were performed until bidirectional block. In case of absence of PV potentials at the beginning of the procedure we considered the vein was isolated if when a temperature under $-40^{\circ} \mathrm{C}$ during at least 120 seconds was reached.

In those patients in AF after PVI, an electrical cardioversion was performed, at the end of the procedure. All patients were maintained under oral anticoagulation for at least two months after the procedure, and $\mathrm{AAD}$ were maintained during the blanking period (three months for paroxysmal and six months for persistent AF).

\section{Follow-up}

After discharge, patients were scheduled for FU visits at 1, 3, 6, 12 and 24 months, with at least four $24 \mathrm{~h}$ Holter monitor recordings (one during the blanking period, a second after AAD withdrawal, a third at 12 months and the last at 24 months). If no recurrences were detected during this time the decision of prolonging the FU was left to discretional decision of the treating doctor. Eighteen patients had an implantable loop recorder or pacemaker with atrial leads. Recurrences during the blanking period were not considered following the consensus publish in 2017(7). After this period, an AF recurrence was considered as documented atrial tachycardia lasting more than 30 seconds.

For the present analysis symptomatic recurrences, new AAD prescription, and weight evolution (to recalculate BMI) were collected by phone interview in all the patients from September 2020 to January 2021. In addition, the Shared Clinical History from the Catalan health system was reviewed in order to complement information regarding arrhythmia recurrence and new AAD prescription during the FU. Finally, their general practitioner obtained a recent EKG in all the patients.

\section{Statistical analysis}

Categorical variables are expressed as absolute and relative frequencies. Continuous variables are expressed as mean $\pm \mathrm{SD}$ or median and range as appropriate. Patients were divided in two groups. Group 1 included patients with a FU longer than 5 years, without recurrences. Group 2 included patients with any recurrence, regardless of FU time. A univariate analysis was performed between both groups, for categorical variables using the $\chi 2$ de Pearson or the Fisher's exact test when appropriate. For continuous variables, the $t$ student test was used.

A multivariate logistic regression analysis using a backward modeling with significant variables $(\mathrm{p}<0.05)$ was performed, to determine prognostic factors of absence of AF recurrence (group 1). Variables were removed one by one, if their exclusion did not modify significantly the likelihood ratio statistics of the model. When removal of any variable changed the estimated parameters of the remaining variables by $>15 \%$, it was considered a confounding effect and that variable was retained in the model regardless of its statistical significance. Results are presented as odds ratio (OR) or $95 \%$ confidence interval (95\% CI). Area under the ROC curve (AUC) was calculated for this model.

Significant continuous variables from EKG were divided in tertiles and Kaplan-Meier curves were performed in order to evaluate the best cut off point predicting the absence of AF recurrence. Those cut off points were included in a logistic regression to obtain the best predictive value for each variable independent or as a whole adjusted by sex and age.

A two-tailed probability value $\leq 0.05$ was deemed significant. Statistical analysis were conducted using the SPSS software v 25.9 (SPSS inc. Chicago. Illinois. USA).

\section{Results}

A total population of 422 patients underwent PVI in our center during this period, nevertheless 21 were lost in the FU, 30 were 


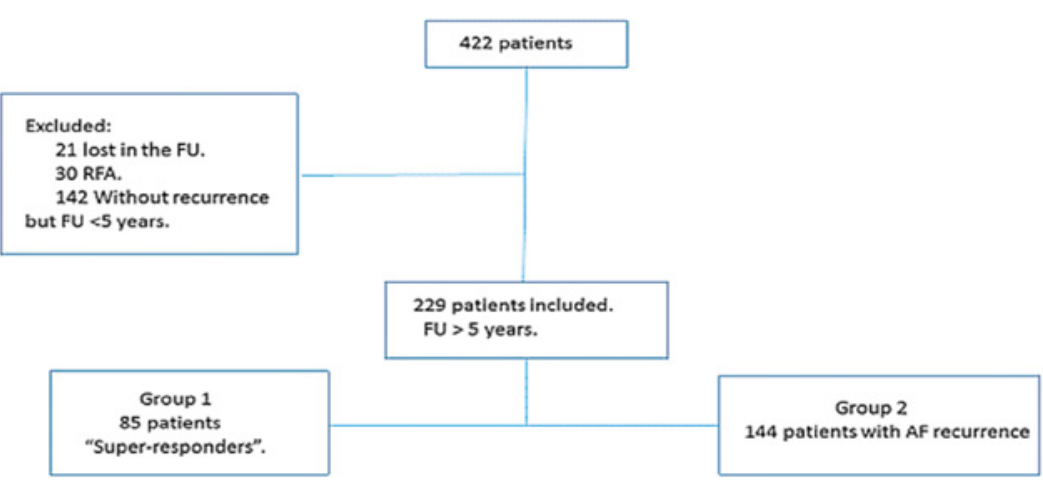

Figure 1: Flowchart of the study. FU: Follow-Up; RFA: Radiofrequency Ablation; AF: Atrial Fibrillation.

2A

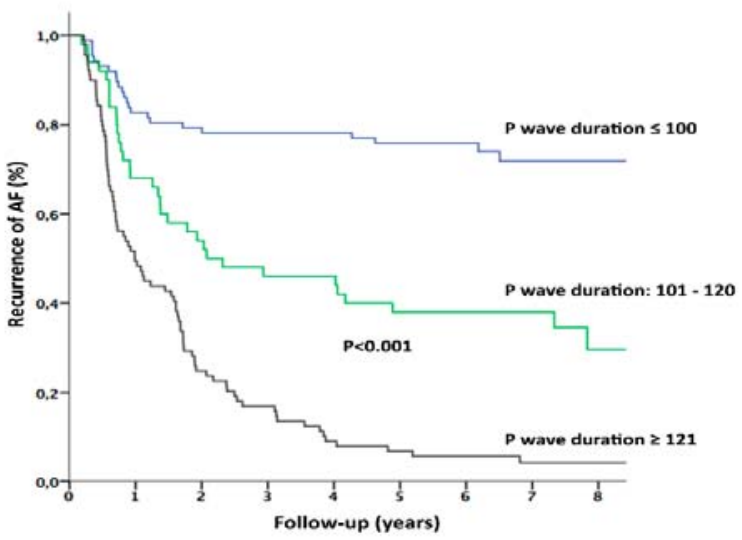

2B

Body mass index

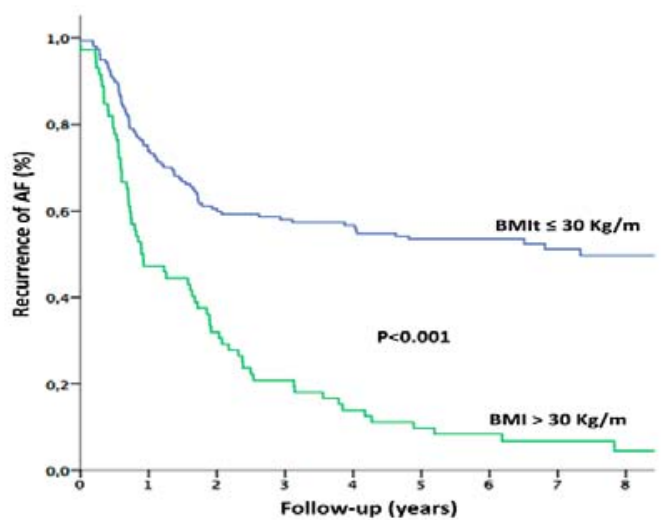

Figure 2: A) Kaplan-Meier (KM) curve for $p$ wave duration. B) KM curve for BMI $(>/<30)$.

excluded because the ablation was done by a point-by-point RFA. Finally, 142 patients had not reached the minimum 5 years FU period. Therefore, for the final analysis we got 229 patients. Figure 1 shows the flowchart of the study.

There were 85 patients, with a median FU of 6.94 (5.77-8.12) years, in whom no AF recurrence was documented. This group was designed as "super-responders" (Group 1) and was compared with the 144 patients with AF recurrences (Group 2).

We didn't find PV potentials in $26 \%$ of the veins. The median time to recurrence was 10.69 (6.69-22.74) months, being significantly shorter in persistent vs paroxysmal AF 6.90 (2.79-8.08) vs 12.06 (6.7725.23) months ( $\mathrm{p}=0.003)$.

We missed the EKG information in three patients in whom no SR was recorded at anytime. One patient with moderate OSA was treated with Continuous Positive Airway Pressure (CPAP) for other concomitant comorbidities, at the same time one patient with severe OSA was not treated for intolerance to CPAP. Only seventeen patients showed a weight decrease achieving a BMI under $30 \mathrm{~kg} / \mathrm{m}^{2}$, among them three summited to bariatric surgery.

We did not find differences between Groups 1 and 2 by using the $\mathrm{CB} 1$ or $\mathrm{CB} 2$. This is probably due to a selection bias. CB1 was initially used mainly in paroxysmal forms of AF, while with $\mathrm{CB} 2$ we performed also in persistent and long-standing AF. Even knowing that only PVI will be not enough in these patients, it was used as a first approach, advising patients that probably a second approach will be needed in the future.

Table 1 shows clinical, echocardiographic, electrocardiographic differences and those related with the procedure in both groups. Univariate analysis showed that patient's in group 1 were younger; hypertension rate, $\mathrm{CHA}_{2} \mathrm{DS}_{2}$ VASc score, moderate OSA rate, $\mathrm{BMI}, \mathrm{p}$ wave duration, time from diagnosis to ablation and left atrial size, was lower than in group 2. By the other hand LVEF, $\mathrm{p}$ wave amplitude, were higher and the temperature under $-40^{\circ} \mathrm{C}$ was achieved in a greater number of cases in group $1 v s$. group 2.

In a logistic regression multivariate analysis we observed that the factors related with long-term maintenance of SR were the $p$ wave duration, BMI, being in SR the ablation index day and finally achieving a minimal temperature in all PV of less of $-40^{\circ} \mathrm{C}$ (Table 2).

As Figure $2 \mathrm{~A}$ and $2 \mathrm{~B}$ show, a $\mathrm{p}$ wave duration $<100 \mathrm{~ms}$ and maintaining a $\mathrm{BMI}<30 \mathrm{Kg} / \mathrm{m}^{2}$ in the $\mathrm{FU}$, was associated a significant reduction in AF recurrence (Log-rank p <0.001).

From a practical and clinical point of view, those variables that significantly predicted long-term maintenance in SR, individually or in combination, either if the minimal temperature lower than $-40^{\circ} \mathrm{C}$ 
Table 1: Univariate analysis comparing Group 1 vs. Group 2.

\begin{tabular}{|c|c|c|c|}
\hline Clinical Variables & $\begin{array}{c}\text { Group } 1 \\
(n=85)\end{array}$ & Group $2(n=144)$ & $\mathbf{P}$ \\
\hline Age & $53.01 \pm 9.81$ & $55.47 \pm 9.90$ & 0.07 \\
\hline Male Sex & $65(76.5 \%)$ & $98(68.1 \%)$ & 0.174 \\
\hline HTA & $27(31.8 \%)$ & $88(61.1 \%)$ & $<0.001$ \\
\hline DM & $6(7.1 \%)$ & $19(13.2 \%)$ & 0.15 \\
\hline CAD & $2(2.4 \%)$ & $8(5.6 \%)$ & 0.252 \\
\hline $\mathrm{CHA}_{2} \mathrm{DS}_{2}$ VASc score & $0.96 \pm 1.09$ & $1.42 \pm 1.14$ & 0.003 \\
\hline OSA & & & $>0.001$ \\
\hline Severe OSA (CPAP) & $7(8.2 \%)$ & $12(8.3 \%)$ & \\
\hline Moderate OSA & $4(4.7 \%)$ & $56(38.9 \%)$ & \\
\hline No or Mild OSA & $13(15.3 \%)$ & $19(13.2 \%)$ & \\
\hline PSG Not performed & $61(71.7 \%)$ & $57(39.6 \%)$ & \\
\hline Initial BMI & $27.02 \pm 3.95$ & $29.61 \pm 4.33$ & $>0.001$ \\
\hline Final BMI & $26.86 \pm 3.93$ & $29.87 \pm 4.35$ & $<0.001$ \\
\hline Final BMI <30Kg/m² & $81(95.3 \%)$ & $76(52.8 \%)$ & $<0.001$ \\
\hline $\begin{array}{l}\text { Time Diagnosis-Ablation } \\
\text { (months)* }\end{array}$ & $24(12.06-48.3)$ & $\begin{array}{l}34.28(18.78- \\
66.28)\end{array}$ & 0.03 \\
\hline Type of AF & & & $<0.001$ \\
\hline Paroxysmal AF & $80(94.1 \%)$ & $96(66.7 \%)$ & \\
\hline Persistent AF & $4(4.7 \%)$ & $33(22.9 \%)$ & \\
\hline Long standing persistent AF & $1(1.2 \%)$ & $15(10.4 \%)$ & \\
\hline \multicolumn{4}{|l|}{ Echocardiographic Variables } \\
\hline LA Size $(\mathrm{mm})$ & $37.78 \pm 4.69$ & $42.66 \pm 4.83$ & $<0.001$ \\
\hline LVEF (\%) & $64.60 \pm 6.17$ & $61.40 \pm 8.37$ & $<0.001$ \\
\hline Electrocardiographic Variables & $\begin{array}{c}\text { Group } 1 \\
(n=85)\end{array}$ & $\begin{array}{c}\text { Group } 2 \\
(n=141)^{\star *}\end{array}$ & p \\
\hline P wave amplitude (mv) & $0.124 \pm 0.029$ & $0.103 \pm 0.036$ & $<0.001$ \\
\hline$P$ wave duration (ms) & $98.2 \pm 12.6$ & $121.03 \pm 14.45$ & $<0.001$ \\
\hline Ablation related Variables & $\begin{array}{c}\text { Group } 1 \\
(n=85)\end{array}$ & Group $2(n=144)$ & p \\
\hline SR the ablation index day & $81(95.3)$ & $104(72.2 \%)$ & $<0.001$ \\
\hline $\begin{array}{l}\text { Minimal Temperature }<-40^{\circ} \mathrm{C} \text { in } \\
\text { all PV }\end{array}$ & $69(81.2 \%)$ & $86(59.7 \%)$ & 0.001 \\
\hline $\begin{array}{l}\text { Acute Efficacy (Bidirectional } \\
\text { isolation) }\end{array}$ & $84(98.8 \%)$ & $136(94.4 \%)$ & 0.075 \\
\hline $\begin{array}{l}\text { Normal Anatomy (4 independent } \\
\text { PV) }\end{array}$ & $73(85.9)$ & $116(80.6)$ & 0.305 \\
\hline Associated CTI Ablation & $25(29.4 \%)$ & $52(36.1 \%)$ & 0.3 \\
\hline
\end{tabular}

HTA: Hypertension; DM: Diabetes Mellitus; CAD: Coronary Artery Disease; OSA Obstructive Sleep Apnea; PSG: Polisomnography; BMI: Body Mass Index; AF: Atrial Fibrillation; LA: Left Atrium; LVEF: Left Ventricular Ejection Fraction; PV Pulmonary Veins; SR: Sinus Rhythm; CTI: Cavo-Tricuspidid Isthmus. "Median (interquartile); "*n for the electrocardiographic variables in group 2 was 141 patients because we missed 3 patients in AF in whom a $p$ wave measure was not possible.

in all PV, is achieved or not, are reflected in Figure 3. In this figure, for example, we can observe that the five years probability of maintaining SR is $86 \%$ in a patient that presented in SR the ablation index day, had a $\mathrm{p}$ wave duration $<100 \mathrm{~ms}$ and maintained a BMI under $30 \mathrm{Kg} /$ $\mathrm{m}^{2}$. However, it rise up to $94 \%$ if the ablation index day, the operator is able to achieve a temperature under $-40^{\circ} \mathrm{C}$ in all the $\mathrm{PV}$ during at least 120 s.

\section{Discussion}

The present study shows that in those patients in whom a CBA is

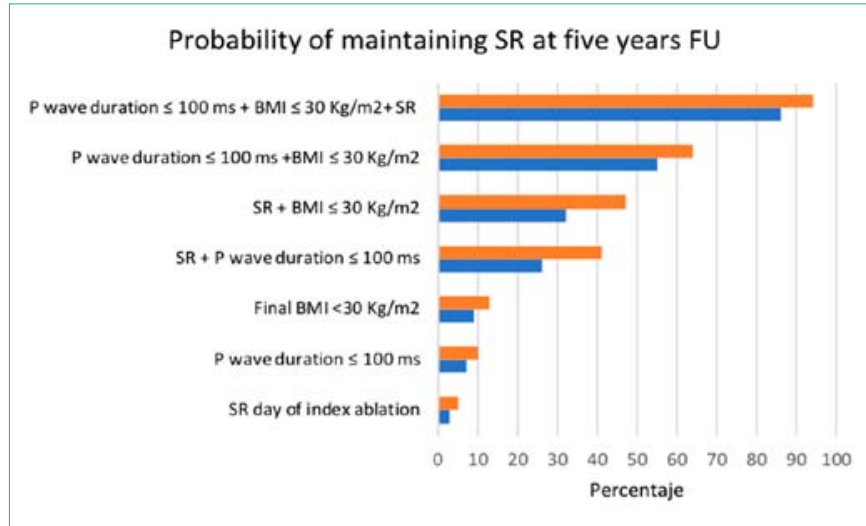

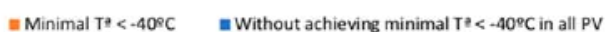

Figure 3: Likelihood of maintaining SR at 5 Years FU after index CBA, using clinical and electrocardiographic variables isolated or in combination, and also adding the possibility of achieving a minimal temperature under $-40^{\circ} \mathrm{C}$ in all PV during the procedure. SR: Sinus Rhythm; BMI: Body Mass Index; PV: Pulmonary Veins; FU: Follow-Up.

Table 2: Logistic regression multivariate analysis, adjusted by age, sex, $p$ wave amplitude, AF type, HTA, LVEF and LA size.

\begin{tabular}{|l|c|c|c|}
\hline \multicolumn{1}{|c|}{ Variable } & OR & $95 \%$ Cl & p \\
\hline Minimal $\mathrm{T}^{\mathrm{a}}<-40^{\circ} \mathrm{C}$ in all PV & 3.52 & $1.45-8.54$ & 0.005 \\
\hline P wave duration & 0.92 & $0.89-0.94$ & $<0.001$ \\
\hline SR the CB index ablation day & 7.29 & $1.53-34.71$ & 0.012 \\
\hline BMl Kg/m ${ }^{2}$ & 0.74 & $0.65-0.85$ & $<0.001$ \\
\hline
\end{tabular}

The AUC and ROC curve was 92\% (95\% Cl: 89-96); p >0.001. PV: Pulmonary Veins; BMI: Body Mass Index; CB: Cryoballoon.

planned, some simple and feasible clinical and EKG variables allow to select a subgroup of patients that will have an excellent behavior in the long-term FU. Protector factors were having a $\mathrm{p}$ wave duration $<100 \mathrm{~ms}$, a maintained BMI $<30 \mathrm{~kg} / \mathrm{m}^{2}$ and the presence of SR the ablation index day. The other protective factor found in this study was to achieve a minimal temperature less than $-40^{\circ} \mathrm{C}$ in all the PV the CBA day, despite this factor cannot be chosen by the referent clinician.

Obesity, is associated with an electrical and structural atrial remodeling and has been clearly related to the appearance and progression of AF from paroxysmal to persistent forms, and has also been related to an increase of recurrences after AF ablation $[5,8]$. Moreover, ablation alone does not stop AF progression in obese patients $[9,10]$. In addition, weight loss has been related to a reduction of left atrial size, left ventricular hypertrophy and even with a reduction of symptoms and AF burden [11]. Indeed weight loss is associated with better post ablation results. In the REVERSE $\mathrm{AF}[12]$ a $10 \%$ weight loss was associated with a long-term reduction of AF progression, with a significant decrease of AF burden or even with complete disappearance of AF. Similar conclusions were observed in the Winkle et al. [10] study on 2715 patients summited for AF ablation; in this study, a higher BMI was associated with more comorbidities and more long standing persistent AF forms. Indeed a BMI $>35 \mathrm{~kg} / \mathrm{m}^{2}$ was associated with worst outcomes.

In our series, although been a retrospective study, which did not include an specific intervention program, we were able to demonstrate that patients, with a maintained $\mathrm{BMI}<30 \mathrm{~kg} / \mathrm{m}^{2}$ or with an initial BMI 
$>30 \mathrm{~kg} / \mathrm{m}^{2}$, but who showed a weight reduction during $\mathrm{FU}$, achieving a BMI $<30 \mathrm{~kg} / \mathrm{m}^{2}$, were most likely to keep SR, than those in whom BMI was maintained over $30 \mathrm{~kg} / \mathrm{m}^{2}$.

$\mathrm{P}$ wave represents atrial depolarization and the endo-epicardial sinus wave propagation over natural barriers [13]. An increase of $p$ wave duration reflects the presence of some kind of atriopathy or atrial myocardiopathy [14] may be favored for multiple risk factors as age, hypertension, coronary artery disease and obesity. In this sense is not surprising, that an increase of $\mathrm{p}$ wave duration had been related to an increase of AF prevalence $[15,16]$, probably reflecting that AF is the final step of the atrial myocardiopathy. Mugnai P et al. [17] in a series of 426 patients after PVI, excluding those with left atrial dilatation, patients with pacemakers or patients in whom the EKG quality was not good enough, observed that $\mathrm{p}$ wave duration and dispersion were clearly related to an increase of AF recurrences after PVI, in patients with normal atrial size.

In our series, a $\mathrm{p}$ wave duration $<100 \mathrm{~ms}$ is a protective factor for no $\mathrm{AF}$ recurrence in the long-term $\mathrm{FU}$, probably due to the fact, that those patients had not a substrate favoring AF, and reflecting that may be a focal trigger in PV was the driver of their AF. Suggesting at the same time that an early treatment on these kind of patients may be justified to avoid progression to atrial myocardiopathy.

Achieving a minimal temperature under $-40^{\circ} \mathrm{C}$ in all $\mathrm{PV}$ was a predictive factor of maintenance of SR in the long-term FU. To achieve that temperature in a single vein has been taken in our study as a surrogate of efficacy, when PV potentials were not recorded in the inner lumen catheter. Nevertheless efficacy between both groups was not different (indeed in most patients PVI was recorded with temperatures $<-40^{\circ} \mathrm{C}$ ). Therefore, in our opinion, achieving less than $-40^{\circ} \mathrm{C}$ in all the PV may reflect a better PV occlusion with deeper or even more extensive lesions in order to avoid recurrence of conduction from the vein to the atria, which is usually the described mechanism of AF relapses [18].

In the study from Watanabe $\mathrm{R}$ et al. [19] in 130 patients who underwent $\mathrm{CBA}$, a temperature $<-44^{\circ} \mathrm{C}$ was a predictor of recurrence at 13 months FU period. However, to our knowledge there are not studies validating if achieving a minimal temperature of $-40^{\circ} \mathrm{C}$ in all $\mathrm{PV}$ is a predictor of recurrence in the long term FU.

The presence of SR the ablation index day, was reflecting in most cases patients with paroxysmal forms (only two patients with paroxysmal AF were in AF the ablation index day), nevertheless up to 18 patients with persistent AF were in SR the ablation index day, after an electrical cardioversion, performed several weeks before underwent CBA. The fact of maintaining SR in these persistent forms after cardioversion, might reflects that those patients had a not so ill atrium. Indeed a correlation of a higher recurrence rate after electrical cardioversion was observed in patients with a HATCH score $>2$ [20] (hypertension, age, chronic obstructive pulmonary disease, stroke and heart failure), suggesting more atriopathy in this group of patients. In another study, a worst atrial myocardial strain was able to predict future recurrences after cardioversion [21].

Despite having statistical significance in the univariate analysis for maintenance of SR, a $\mathrm{p}$ wave amplitude greater than $0.13 \mathrm{mv}$ lost it significance in the multivariate analysis. This fact probably happened because the population included in our series was quite "healthy". $\mathrm{P}$ wave amplitude may correlate with endocardial voltage, and probably the more substrate in the atrium, the lower $\mathrm{p}$ wave amplitude. Park JK et al. [22], in a series of more than 500 patients who underwent PV ablation showed that a $\mathrm{p}$ wave amplitude $<0.1 \mathrm{mv}$ in lead DI was a predictive factor of recurrence.

Moderate OSA, obviously not treated with CPAP, did not reach statistical significance in the multivariate, although it did, in the univariate analysis, may be due to the fact of confounding factors as obesity [23], or just due to the small number of patients included in this series. Nevertheless, non-treated severe OSA is a known factor related with an increase of AF recurrences after PVI, and treatment with CPAP in this kind of patients decreased recurrences [24]. Is interesting to highlight that sleep units carried out by pneumologists are not treating moderate OSA with CPAP, and they seem to have a higher risk of recurrence.

\section{Conclusion}

In our series having a p wave duration $<100 \mathrm{~ms}, \mathrm{BMI}<30 \mathrm{~kg} / \mathrm{m}^{2}$, the presence of SR the ablation index day and achieve a minimal temperature under $-40^{\circ} \mathrm{C}$ in all $\mathrm{PV}$ for at least 120 s during $\mathrm{CBA}$ are associated with maintenance of SR in the long-term FU. A holistic treatment in AF patients, taking specifically care on decrease weight may improve outcomes. An appropriate selection of patients undergoing CBA can improve outcomes and resource optimization.

\section{Limitations}

Several limitations are present in this study. The main limitation of the present study is being a retrospective study, including patients with CBA with CB1 and CB2 and also with different application time per vein. Nevertheless, there were no differences in efficacy because of that issue. The second point is that this is not an interventional study, and this is the reason that weight loss was achieved in only 17 patients with an initial $\mathrm{BMI}>30 \mathrm{~kg} / \mathrm{m}^{2}$, but even with this limitation, this variable appears as significant. The third point is that we do not have polysomnography in all the patients, and may be more patients with recurrences were non-detected OSA. The forth point is that we do not have study the atrial function by atrial myocardial strain, and this point is especially important in patients with recurrences and normal atrial size. Previous studies showed a correlation between atrial strain and $\mathrm{p}$ wave duration 25 . The fifth point is that we measured the $\mathrm{p}$ wave duration after CBA, once the ADD had been withdrawn. The reason to do it this way, was to avoid the effect of these drugs (only in four patients we used the ECG just after ablation, so under the effect of $\mathrm{AAD}$, because in the first clinical control they were in $\mathrm{AF}$ ). By the other hand, it should be impossible to measure the $\mathrm{p}$ wave in patients with persistent $\mathrm{AF}$, except if they had been previously cardioverted. However, doing it after ablation can be also discussed, by the fact that CBA could have influence the measure. In order to discard this possibility we measured the $\mathrm{p}$ wave amplitude and duration in 50 patients ( 25 paroxysmal and 25 persistent) pre and post ablation both under the effect of $\mathrm{AAD}$, and no significant differences between the values was observed. Finally, because of the retrospective nature of this study, not having continuous monitoring on heart rhythm with any device (only in 18 patients), may not discard asymptomatic AF recurrences. However, the Shared Clinical History of the Catalan Health system allowed us to review all the medical history of the 
patients during $\mathrm{FU}$, and we reassure that none "super-responder" patient received $\mathrm{AAD}$ during the $\mathrm{FU}$ time, and of course, neither the general practitioner nor cardiologist reported any recurrence.

\section{Highlight Points}

- After a long term follow up period some patients summited for cryo-balloon ablation either for paroxysmal or persistent atrial fibrillation remain in sinus rhythm. Characterize this kind of patients is the aim of the present study.

- $\quad$ Some simple and feasible clinical and EKG variables allow selecting a subgroup of patients that will have an excellent behavior in the long-term follow-up.

- A p wave duration $<100 \mathrm{~ms}$, a maintained BMI $<30 \mathrm{~kg} /$ $\mathrm{m}^{2}$ and the presence of SR the ablation index day and a minimal temperature less than $-40^{\circ} \mathrm{C}$ in all the pulmonary veins were related with better outcomes.

\section{References}

1. Hindricks G, Potpara T, Dagres N, Arbelo A, Bax JJ, Blomström-Lundqvist C, et al. 2020 ESC guidelines for the diagnosis and management of atrial fibrillation developed in collaboration with the European Association of Cardio-Thoracic Surgery (EACTS). Eur Heart J. 2020.

2. KH Kuck, J Brugada, A Fürnkranz, A Metzner, F Ouyang, KRJ Chun, et al Cryoballoon or Radiofrequency Ablation for Paroxysmal Atrial Fibrillation. NEJM. 2016; 374: 2235-2245

3. E Hoffmann, F Straube, K Wegscheider, M Kuniss, D Andresen, Li-Qun Wu et al. Outcomes of cryoballoon or radiofrequency ablation in symptomatic paroxysmal or persistent atrial fibrillation. Europace. 2019; 21: 1313-1324.

4. RR Tilz, CH Heeger, A Wick, AM Saguner, A Metzner, A Rillig, et al. Ten-Year Clinical Outcome After Circumferential Pulmonary Vein Isolation Utilizing the Hamburg Approach in Patients With Symptomatic Drug-Refractory Paroxysmal Atrial Fibrillation. Circ Arrhythm Electrophysiol. 2018; 11 e005250.

5. G Mugnai, G Paparella, I Overeinder, E Ströker, J Sieira, A Bisignani, et al. Long-term clinical outcomes after single freeze cryoballoon ablation for paroxysmal atrial fibrillation: a 5-year follow-up. J. Interv Card Electrophysiol. 2021.

6. WH Sauer, ML McKernan, D Lin, EP Gerstenfeld, DJ Callans, FE Marchlinski. Clinical predictors and outcomes associated with acute return of pulmonary vein conduction during pulmonary vein isolation for treatment of atria fibrillation. Heart Rhythm. 2006; 3: 1024-1028.

7. Calkins H, Hindricks G, Cappato R, Kim YH, Saad EB, Aguinaga L, et al. HRS/EHRA/ECAS/APHRS/SOLAECE expert consensus statement on catheter and surgical ablation of atrial fibrillation. Heart Rhythm. 2017; 14: e275-e444.

8. Pathak RK, Middeldorp ME, Meredith M, Mehta AB, Mahajan R, Wong CX, et al. Long-Term Effect of Goal-Directed Weight Management in an Atria Fibrillation Cohort: a Long-Term Follow-Up Study (LEGACY). J Am Coll Cardiol. 2015; 65: 2159-2169.

9. Dublin S, French B, Glazer NL, Wiggins KL, Lumley T, Psaty BM, et al. Risk of new-onset atrial fibrillation in relation to body mass index. Arch Intern Med. 2006; 166: 2322-2328.

10. RA Winkle, R Hardwin Mead, G Engel, MH Kong, W Fleming, J Salcedo, et al. Impact of obesity on atrial fibrillation ablation: Patient characteristics, long-term outcomes, and complications. Heart Rhythm. 2017; 14: 819-827.
11. Abed HS, Wittert GA, Leong DP, Shirazi MG, Bahrami B, Middlecorp ME, et al. Effect of weight reduction and cardiometabolic risk factor management on symptom burden and severity in patients with atrial fibrillation: a randomized clinical trial. JAMA. 2013; 310: 2050-2060.

12. ME Middeldorp, RK Pathak, M Meredith, AB Mehta, AD Elliott, R Mahajan, et al. Prevention and regressive effect of weight-loss and risk factor modification on Atrial Fibrillation: the REVERSE-AF study. Europace. 2018; 20: 19291935.

13. D Calvo, D Filgueiras, and J Jalife. Propagation of Sinus Waves in the Atrial Architecture: When Laminar Electrical Fluxes Turn Turbulent. Circ Arrhythm Electrophysiol. 2017; 10.

14. Kamel H, Okin PM, Longstreth WT, Elkind MSV, Soliman EZ. Atria cardiopathy: A Broadened concept of left atrial thromboembolism beyond atrial fibrillation. Future Cardiol. 2015; 11: 323-331.

15. Magnani JW, Zhu L, Lopez F, Pencina MJ, Agarwal SK, Soliman EZ, et al. P-wave indices and atrial fibrillation: Cross-cohort Assessments from the Framingham Heart Study and Atherosclerosis Risk in Communities Study. Am Heart J. 2015; 169: 53-61.

16. Nielsen JB, Kühl JT, Pietersen A, Graff C, Lind B, Struijk JJ, et al. P-wave duration and the risk of atrial fibrillation: Results from the Copenhagen ECG Study. Heart Rhythm. 2017; 12: 1887-1895.

17. G Mugnai, GB Chierchia, C de Asmundis, J Juliá, G Conte, J Sieira-Moret, et al. P-wave indices as predictors of atrial fibrillation recurrence after pulmonary vein isolation in normal left atrial size. J Cardiovasc Med. 2016; 17: 194-200.

18. F Ouyang, M Antz, S Ernst, H Hachiya, H Mavrakis, FT Deger, et al Recovered pulmonary vein conduction as a dominant factor for recurrent atrial tachyarrhythmias after complete circular isolation of the pulmonary veins: lessons from double Lasso technique. Circulation. 2005; 111: 127-135.

19. Watanabe R, Okumura Y, Nagashima K, Iso K, Takahashi K, Arai M, et al. Influence of balloon temperature and time to pulmonary vein isolation on acute pulmonary vein reconnection and clinical outcomes after cryoballoon ablation of atrial fibrillation. J Arrhythm. 2018; 34: 511-519.

20. SV Emren, U Kocabaş, H Duygu, F Levent, EC Şimşek, ZY Emren, et al. The role of HATCH score in predicting the success rate of sinus rhythm following electrical cardioversion of atrial fibrillation. Kardiol Pol. 2016; 74: 978-984.

21. G Dell'Era, E Rondano, E Franchi, PN Marino. Atrial Fibrillation (NAIF) Study Group. Atrial asynchrony and function before and after electrical cardioversion for persistent atrial fibrillation. Eur J Echocardiogr. 2010; 11: 577-583.

22. JK Park, J Park, JS Uhm, B Joung, MH Lee, and HN Pak. Low P-wave amplitude $(<0.1 \mathrm{mV})$ in lead $\mathrm{I}$ is associated with displaced inter-atrial conduction and clinical recurrence of paroxysmal atrial fibrillation after radiofrequency catheter ablation. Europace. 2016; 18: 384-391.

23. Chilukuri K, Dalal D, Gadrey S, Marine JE, MacPherson E, Henrikson CA, et al. A prospective study evaluating the role of obesity and obstructive sleep apnea for outcomes after catheter ablation of atrial fibrillation. J Cardiovasc Electrophysiol. 2010; 21: 521-525.

24. Patel D, Mohanty P, Di Biase L, Shaheen M, Lewis WR, Quan K, et al. Safety and efficacy of pulmonary vein antral isolation in patients with obstructive sleep apnea: the impact of continuous positive airway pressure. Circ Arrhythm Electrophysiol. 2010; 3: 445-451.

25. Lacalzada-Almeida J, Izquierdo-Gómez MM, García-Niebla J, Elosua $R$ Jiménez-Sosa A, Baranchuk A, et al. Advanced interatrial block is a surrogate for left atrial strain reduction which predicts atrial fibrillation and stroke. Ann Noninvasive Electrocardiol. 2019; 24: e12632. 